\title{
The Ambivalent Appraisal of Job Demands and the Moderating Role of Job Control and Social Support for Burnout and Job Satisfaction
}

\author{
Joachim Gerich ${ }^{1} \cdot$ Christoph Weber ${ }^{2}$
}

Accepted: 30 September 2019 / Published online: 11 October 2019

(c) The Author(s) 2019

\begin{abstract}
Within an extended challenge-hindrance framework, it is assumed that job demands are subjectively appraised both as challenges (that is, as working conditions that are associated with potential personal gains) and hindrances (as working conditions associated with constrains) at the same time. In accordance with transactional stress theory, the association between demand intensity and work-related attitudes (work satisfaction) and psychological strain (burnout) is expected to be mediated by individual appraisal. Moreover, because curvilinear relationships between demand and challenge and hindrance appraisals are assumed, and appraisal is expected to be moderated by job control and social support, we tested complex nonlinear moderated mediation models for four types of job demands (task difficulty, time pressure, interruptions, and responsibility). Based on cross-sectional data of a heterogeneous sample of employees, we confirmed simultaneous challenge and hindrance appraisals. Challenge components are positively associated and hindrance components are negatively associated with favorable outcomes (higher work satisfaction and lower burnout). Challenge appraisals are found to be more relevant for work satisfaction, while hindrance appraisals are more relevant for burnout. The relationship between demand intensity and challenge appraisal is confirmed as curvilinear, whereas hindrance appraisals are approximately linearly related to demand intensity. The relationship between demand intensity and outcome variables is partly mediated by challenge and hindrance appraisal, and significant interaction effects suggest that the appraisal process is moderated by job control and social support.
\end{abstract}

Keywords Challenge stressors $\cdot$ Hindrance stressors $\cdot$ Stressor appraisal $\cdot$ Job control . Social support

Electronic supplementary material The online version of this article (https://doi.org/10.1007/s1120 5-019-02195-9) contains supplementary material, which is available to authorized users.

Joachim Gerich

Joachim.gerich@jku.at

Christoph Weber

Christoph.weber@ph-ooe.at

1 Department of Sociology, Johannes Kepler University Linz, Altenbergerstr. 69, 4040 Linz, Austria

2 University of Education Upper Austria, Kaplanhofstr. 40, 4020 Linz, Austria 


\section{Introduction}

Over many years, research on occupational stress has proven that working conditions affect the health- and work-related well-being of employees (Sonnentag and Frese 2003; Ganster and Rosen 2013). While much of this research has focused on the negative consequences of work stress, there is growing interest in the ambivalent role of work stressors. Although it has been long recognized that stressors may not only provoke unfavorable effects for individuals [for example, Selye's (1974) concept of eustress and distress], the challenge-hindrance stressor framework (Cavanaugh et al. 2000) has especially stimulated research on the ambivalent nature of stressors. Cavanaugh et al. (2000) suggested differentiating "negative" hindrance stressors from challenge stressors that offer potential gains for employees despite their taxing nature. Based on this stressor classification, those authors found that hindrance stressors are associated with elevated strain and reduced positive work attitudes, whereas challenge stressors are associated with elevated strain but also elevated positive work attitudes (Boswell et al. 2004; Cavanaugh et al. 2000; LePine et al. 2004; Podsakoff et al. 2007). However, other authors have questioned the a priori classification of stressors into challenges and hindrances. For example, Bakker (2014, p. 234) argued that "it is still unclear whether daily work pressure acts as a challenge or hindrance stressor" and that the role of such stressors likely varies for different resources of work environments embedded in distinct occupational sectors. Moreover, recent studies (Gerich 2016; Searle and Auton 2015; Webster et al. 2011) found substantial inter-individual variance in stressor appraisal regarding challenge and hindrance perception. Thus, there are strong indications that stressors might be simultaneously appraised as both a challenge and a hindrance.

The aim of the present study is to analyze the appraisal of four work-related stressors (time pressure, interruptions, task complexity, and responsibility) in a heterogeneous sample of employees, with respect to the dimensions of hindrance and challenge. Time pressure, responsibility, and task complexity were chosen because they are demands that are typically categorized as challenge stressors (Cavanaugh et al. 2000; Webster et al. 2011). In addition, the potential ambivalent nature of interruptions in its challenging and hindering properties has been noted by some researchers (Chong et al. 2011; Jett and George 2003; Zoupanou 2015). Jett and George (2003) differentiated between different types of interruptions (intrusions, breaks, distractions, and discrepancies) and argued that, despite their potential negative effects for organizational members, interruptions may also be beneficial with respect to flow of information or stimulation for boring or routine tasks.

Following an extended stress model which is shown in Fig. 1, and which will be elaborated on the following sections-we examine whether stressor appraisal is affected by two selected work-related resources (perceived social support and job control). We also test whether the association of stressor intensity with health-related (burnout) and work-related (work satisfaction) outcomes is mediated by stressor appraisal and whether this mediation is moderated by perceived social support and job control.

The study proposes a refined general stress model which assumes that job demands affect health- and work-related outcomes through two different (challenge and hindrance) dimensions of stressor appraisal. Moreover, job resources are assumed to moderate the challenge and hindrance appraisal of demands. Contrary to generalized a priori assumptions concerning the nature of job demands as challenges or hindrances, our model assumes that job demands potentially include both dimensions at the same time 


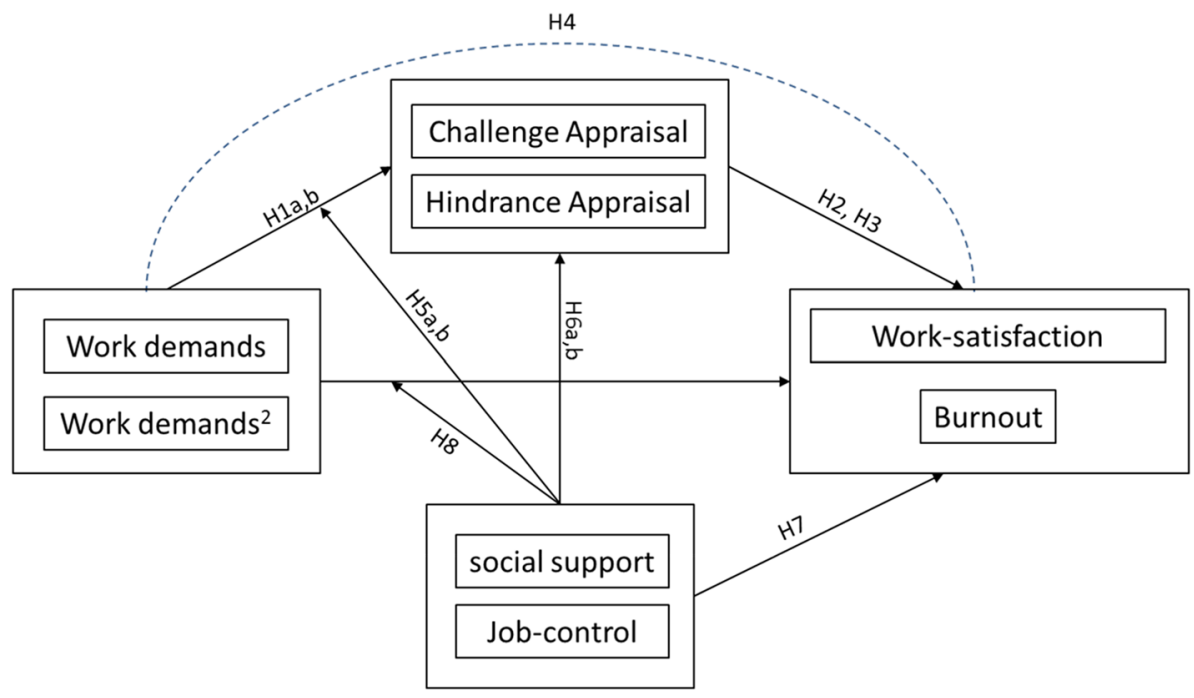

Fig. 1 Conceptual model. Note The conceptual model is analyzed separately for four different work demands (time pressure, task complexity, interruptions, and responsibility). The squared term (work demands ${ }^{2}$ ) is needed to include the assumed curvilinear associations between work demands and appraisal

to varying extents. Hence, the nature of job demands is thought to depend on varying individual evaluations, which can be modified by work-related boundary conditions such as the amount of social support and job control.

\section{The Challenge-Hindrance Framework}

Cavanaugh et al. (2000) criticized the prevailing tendency of stress research to treat the experienced exposure to stressors as a unidimensional construct for the analysis of associations between stressor exposure and work outcomes. Drawing on transactional stress theory, the authors argued that the effect of stressor exposure on various outcomes depends on an individual's stressor appraisal. They asserted that some working conditions, which they called challenge stressors, "although potentially stressful, have associated potential gains for individuals," and defined others (hindrance stressors) as working conditions "that tend to constrain or interfere with an individual's work achievement" (Cavanaugh et al. 2000, p. 68). They used assessments of various stressors to empirically classify stressors as either challenging (such as workload or time pressure) or hindering (such as role ambiguity or role conflict). Based on this classification, they found, for a sample of US managers, that exposure to challenge stressors was positively related to work satisfaction and negatively related to job search, whereas exposure to hindrance stressors was negatively related to work satisfaction and positively related to job search. Further research confirmed these results (Boswell et al. 2004; LePine et al. 2004; Pearsall et al. 2009; Podsakoff et al. 2007) with regard to other work-related outcome variables (work satisfaction, withdrawal behavior, turnover intentions, loyalty, team performance, and motivation to learn). Moreover, Boswell et al. (2004) supported their hypothesis that the relation between challenge stress and positive work outcomes was mediated by the perceived job challenge. Contrary to work-related outcome variables, 
however, Boswell et al. (2004) and LePine et al. (2004) found that exposure to both challenge and hindrance stressors was positively related to psychological strain (anxiety and emotional exhaustion), which led them to conclude that although challenge stressors may encourage positive attitudes, exposure to stress leads to strain regardless of the type of stressor.

With respect to general well-being (positive attitude toward life), Widmer et al. (2012) argued that challenge stressors have positive and negative components simultaneously. They found that time pressure (as a challenge stressor) affects well-being positively through self-esteem but negatively through strain. Thereby, negative and positive effects cancel each other out.

Some authors (Chong et al. 2011; Gerich 2016; Searle and Auton 2015; Webster et al. 2011) have contended that an a priori classification of stressors as either challenging or hindering is not in accordance with the assumptions of transactional stress theory, because such classification does not account for the personal and situational variance of stressor appraisal and because some stressors may be appraised as a challenge and a hindrance at the same time. Webster et al. (2011) applied separate challenge and hindrance appraisal measures for four work stressors (workload, responsibility, role conflict, and role ambiguity) and found that all stressors (except responsibility) were positively correlated with challenge and hindrance appraisal, which meant that a stressor could simultaneously be appraised as a challenge and a hindrance. They hypothesized that hindrance appraisal and challenge appraisal were positively and negatively related to strain, respectively. While they confirmed positive relations between hindrance appraisal and psychological and physical strain, they did not find negative relations between challenge appraisal and strain. Contrary to expectations, the challenge appraisal of workload and responsibility was positively related to physical strain. Gerich (2016) reported similar results, but he found that each challenge appraisal for nine stressors was negatively correlated with burnout and physical symptoms and each hindrance appraisal was positively correlated. The different results may be attributable to a difference in the measurement of challenge appraisal. Whereas Webster et al.'s (2011) question on challenge appraisal expressed an attenuation of hindrance (although "potentially stressful, something you think you can overcome"), the notion of personal gain was more pronounced in Gerich's (2016) question of whether a working condition was perceived as a "beneficial opportunity or challenge". Searle and Auton (2015) applied multiple item measures of challenge and hindrance appraisal and confirmed indirect effects of opposite direction for stressors via challenge and hindrance appraisal on work-related affective states. Based on their research, they concluded that separate challenge and hindrance appraisal measures were merited because "even after accounting for the effects of stressors, challenge and hindrance appraisals consistently explained unique variance in affective states" (Searle and Auton 2015, p. 121). Similarly, using a two-dimensional structure of different modalities of time pressure, Chong et al. (2011) found that team coordination and team performance were positive affected by challenge time pressure and negatively affected by hindrance time pressure.

LePine et al. (2016) concluded that although the challenge-hindrance framework is important to understanding differential stressor effects, research was needed regarding the appraisal process with respect to the perceived hindrance and challenge of job demands: "More specifically, we could potentially identify factors that moderate the role of appraisals as transmitter of stressor effects, and, in the end, significantly impact how stressors influence outcomes" (LePine et al. 2016, p. 1037). 


\section{Curvilinear Relationships Between Stressor Intensity and Stressor Appraisal}

Although most research assumed linear associations between stressor intensity and wellbeing (Meyerding 2015; Preston 2018), there are reasons to consider a curvilinear nature of these associations.

In an analogy to vitamin intake, Warr (1994) suggested a "vitamin model" of job demands, which assumes that some job demands are beneficial up to a certain degree. Beyond this threshold, however, either no additional benefits of demanding job characteristics (analogous to vitamins $\mathrm{C}$ and $\mathrm{E}$, this is called CE for "constant effect") or even detrimental health effects for very high levels of demand (as with vitamins $A$ and $D$, this is called AD for "additional decrement") are expected.

The main idea behind the vitamin model is the assumption that there are optimal levels for most job characteristics which are not necessarily identical to maximum or minimum levels. Warr argues that some job features may turn their character from an "opportunity" associated with moderate levels of intensity to an "unavoidable requirement" associated with very high levels of intensity. Too low complexity of job demands, for example, may lead to underload and boredom whereas high levels of complexity leads to overload when it exceeds personal capacities (Warr 2014).

Similarly, activation theory (Gardener and Cummings 1988) assumes that demanding situations (such as time pressure or task complexity) cause a higher level of activation. However, as individuals tend to maintain a characteristic level of activation, an inverted $\mathrm{u}$-shaped relationship between activation and positive affect is expected. If the levels of demand are too low, this causes understimulation and boredom; if they are too high, this provokes an undesired overload.

Chong et al. (2011) and Reis et al. (2016) also determined that previous research on the association between time pressure and work performance, as well as work engagement, was inconclusive by revealing positive, null, and negative associations. Among other explanations for these varying results, the authors proposed that curvilinear relations between time pressure and work engagement were possible reasons for this inconsistency. Based on a diary study, Reis et al. (2016) confirmed an inverted u-shaped relationship between daily levels of time pressure and vigor as perceived by employees. However, as those authors focused on intrapersonal levels of time pressure with person-centered variables, they noted that the optimal level of time pressure may depend upon the employees' individual preferences. Following this argument, one could assume that the curvilinear relationship found in their intrapersonal study between time pressure and perceived vigor could alternatively be attributable to a curvilinear relationship between time pressure and individual stressor appraisal. Given their results, Reis et al. (2016) proposed that future studies should consider curvilinearity and individual appraisal of other challenge demands, such as qualitative workload, job responsibility, and task variability.

With respect to the challenge-hindrance framework of job demands, some authors have speculated that an inverted u-shaped relationship between stressor intensity and positive affect may only apply to challenge stressors and not to hindrance stressors. In their meta-analysis regarding challenge and hindrance stressors (which was based on a priori classifications of stressors), LePine et al. (2005) were not able to analyze curvilinear relationships between challenge and hindrance stressors with outcomes such as motivation, performance, and strain. However, they argued that hindrance stressors (such as role conflict or supervisor-related stress) do not offer any positive opportunities for employees-an 
argument that stands "in sharp contrast" to the idea "that up to some point, all types of stressors at work are good" (LePine et al. 2005, p. 770). Instead, following these arguments, an inverted u-shaped relationship between stressor intensity and outcomes such as positive affect or performance could be expected for challenge stressors but not for hindrance stressors (Leung et al. 2011).

By applying this idea to an extended challenge-hindrance framework, which assumes that stressors are appraised as challenges and hindrances to varying extents at the same time, one could expect a curvilinear relationship between stressor intensity and challenge appraisal, but a positive linear association between stressor intensity and hindrance appraisal. This is expected because an intensification of any work demand is associated with additional effort and depletion of energy, which will be likely appraised as a hindering component. At the same time and despite this hindering aspect, an optimal moderate level of stressor intensity may provide some personal opportunities. Contrary to hindrance appraisal, a curvilinear association between demand intensity and challenge appraisal could be expected. This is because, on one hand, very low levels of job demands are not able to provide any additional opportunities and, on the other hand, very high levels of job demands are likely to deviate from an individual's characteristic level of activation. Therefore, the challenge appraisals of low and high levels are expected to be lower than medium levels of job demands, resulting in an inverted u-shaped association between demand intensity and challenge appraisal (additional decrement; Warr 1994). Apart from this line of arguments, there is also the possibility that increasing job demands lead to higher challenge appraisal up to a certain threshold, but beyond this threshold, increasing demands provide no additional benefits (constant effect; Warr 1994).

This leads to the following hypotheses:

H1a There is a curvilinear relationship of demand intensity regarding time-pressure, responsibility, task-complexity, and interruptions with the challenge appraisal of these demands.

H1b Demand intensity regarding time-pressure, responsibility, task-complexity, and interruptions is positively linear related to hindrance appraisal.

Moreover, following the challenge/hindrance model and transactional stress theory it is expected that the association of work demands with health-related and work-related outcomes is partially mediated by the challenge and hindrance appraisal of these demands, leading to the following hypotheses:

H2 Challenge appraisals of work demands regarding time-pressure, responsibility, taskcomplexity, and interruptions are positively related to work satisfaction and negatively related to burnout.

H3 Hindrance appraisals of work demands regarding time-pressure, responsibility, taskcomplexity, and interruptions are negatively related to work satisfaction and positively related to burnout.

H4 The association between demand intensity regarding time-pressure, responsibility, task-complexity, and interruptions and work satisfaction and burnout is (partially) mediated by stressor appraisal. 
As expressed in Hypothesis H4, the mediation might be partial, which can be expected from prior research, where direct effects between stressors and health-related outcomes were still confirmed when analyses were adjusted for stressor appraisal (Gomes et al. 2016; Webster et al. 2011), leading to the conclusion that working conditions can also be harmful for health regardless of their subjective evaluation. Therefore, a direct path between the exposure to work demands and the outcome variables is included in the conceptual model (Fig. 1).

\section{Control and Support as Moderators of Stressor Appraisal}

The variability of stressor appraisal may be explained by differences in the personal characteristics of employees, such as negative affectivity or self-efficacy (Mackey and Perrewé 2014). Task-related and organizational characteristics can also form boundary conditions that affect how employees appraise specific job demands. Although numerous conditions at the task or organizational level can be considered as such moderators of the stressor appraisal process, job control and social support are two prominent concepts of occupational research that are believed to affect the stress process.

Job control encompasses job characteristics in terms of the employees' opportunities to determine how tasks are done and their decision-making authority regarding timing, location, or method. Job control plays a key role in Karasek's (1979) Job Demand-Control Model (JDC), in which "high strain jobs" as a combination of high demands and low control are associated with the highest risk for illness and impaired wellness, and "low strain jobs" as a combination of low demands and high control are associated with favorable health and well-being. Contrary to high strain jobs, "active" jobs-defined by the combination of high demands and high job control—are expected to be associated with increased motivation, learning, and personal growth. Therefore, it is argued that despite their taxing nature, high demands may be beneficial for motivation and job satisfaction under the condition of high job control. In a subsequent extension of the JDC Model, social support was added as a third key variable in the Job Demand-Control-Support Model (Johnson and Hall 1988).

The Job Demands-Resources (JDR) model, proposed by Bakker and Demerouti (2007), is a more generalized model that extends the assumptions of the JDC model to a wider range of job resources, including dimensions of control such as autonomy, participation in decision-making, and supervisor and coworker support.

These models include hypotheses regarding the main effects of demands and resources as well as the interaction effects of demands and resources for outcome variables such as work-related attitudes and health-related well-being. Whereas previous research confirmed strong evidence for the main effects of control and support for different dimensions of wellbeing, studies have only found weak evidence for the assumed buffer effects (Häusser et al. 2010). However, most research on the buffer effects of resources such as control and support assumed a priori classifications of job stressors. In response, Mackey and Perrewé (2014) suggested that research should consider how job resources (such as job control and social support) affect stressor appraisal as a precursor of the strain process.

Similarly, Spector (2002) proposed that job resources such as control can affect the occupational stress process at different stages. In addition to the possibility that job resources may buffer the effects of stressor exposure on health and well-being, he argued that job resources may modify how the work environment is perceived as a stressor, 
how perceived stressors provoke negative emotions, and how negative emotions lead to strain. Demerouti and Bakker $(2011$, p. 3) further asserted that buffering resources "can reduce the tendency of organizational properties to generate specific stressors, alter the perceptions and cognitions evoked by such stressors, moderate response that follow the appraisal process, or reduce the health-damaging consequences of such responses". Hence, previous research has predominately focused on the possible buffer effects of job resources for the relationship between stressor exposure and outcome variables, but has neglected possible buffer effects for the relationship between stressor exposure and stressor appraisal as a mediator between stressor exposure and outcome variables.

Following these arguments, moderator and main effects of social support and job control for the appraisal process are expected, leading to the following set of hypotheses:

H5a The relationship of demand intensity regarding time-pressure, responsibility, taskcomplexity, and interruptions with challenge appraisal is moderated by social support and job control, such that demand intensity is more strongly appraised as challenge under conditions of high support and high job control.

H5b The relationship of demand intensity regarding time-pressure, responsibility, taskcomplexity, and interruptions with hindrance appraisal is moderated by social support and job control, such that demand intensity is more strongly appraised as hindrance under conditions of low support and low job control.

It should be noted that these expected interaction effects in combination with the assumed mediation effects (as expressed in H4), lead to the assumption of a moderated mediation model. More precisely, following transactional stress theory, it is expected that the effect of the exposure to the four work demands on burnout and job satisfaction is mediated by the challenge and hindrance appraisal of the work demands. However, because the appraisal of work demands is expected to depend on the amount of social support and job control, the resulting mediation path is also conditional on the availability of these two resources.

Moreover, by extending JDC and JDR models with the challenge/hindrance Framework also main-effects of job resources on the appraisal of job demands could be expected, leading to the following hypotheses.

H6a Social support and job control are positively related with the challenge appraisal regarding time-pressure, responsibility, task-complexity, and interruptions, irrespective of demand intensity.

H6b Social support and job control are negatively related with the hindrance appraisal regarding time-pressure, responsibility, task-complexity, and interruptions, irrespective of demand intensity.

In addition however, and irrespective of possible appraisal paths, job resources may operate in accordance with the original assumptions drawn by the JDC or the JDR model. That is, job control and social support may have a direct positive main effect and a moderation effect on wellbeing, irrespective of the assumed appraisal mechanisms. This leads to the following hypotheses: 
H7 Job control and social support are directly related to higher work satisfaction and reduced burnout.

H8 The direct effects of job demands regarding time-pressure, responsibility, task-complexity, and interruptions on work satisfaction and burnout are moderated by job control and social support, such that job demands are associated with more desired outcomes under condition of high job control and social support.

\section{Methods}

\subsection{Sample}

A standardized questionnaire was mailed to a random sample of 3000 employees covered for health insurance by the Upper Austrian Sickness Fund, the mandatory health insurance provider for all private employees in the region of Upper Austria. Six hundred and thirtyone employees (21\%) responded to the questionnaire. As the parent population of the random sample covers nearly $90 \%$ of employees in Upper Austria, the sample includes a broad variety of occupations. The mean age of respondents is 44.6 years $(\mathrm{SD}=9.7)$ and $48.8 \%$ are female. The sample includes employees with different occupational status ( $12.1 \%$ unskilled workers; $18.3 \%$ skilled workers; $48.1 \%$ professionals; $21.5 \%$ managers), and education (6.0\% primary or lower secondary; $63.2 \%$ vocational education; $18.1 \%$ upper secondary; 12.7 tertiary). Mean tenure is 15.4 years $(\mathrm{SD}=10.6)$.

\subsection{Measures}

Exposure to work-related demands (demand intensity) was measured on a five-point rating format, ranging from "does not apply at all" to "fully applies". The work-related demands covered interruptions ("I am frequently interrupted from my primary work tasks"), time pressure ("I am often under time pressure"), task complexity ("my work covers complicated tasks"), and responsibility ("I have to carry a lot of responsibility in my work"). These items (except for responsibility) are based on items covered by a risk inventory, which is frequently used in German-speaking countries (short questionnaire for job analysis, Prümper et al. 1995).

Each exposure question was followed by challenge and hindrance appraisal questions. The challenge appraisal for each working condition was covered by the statement "I feel that this condition [that is, the previously rated exposure to the working condition] is a beneficial opportunity/challenge for me". The hindrance appraisal for each working condition was covered by the statement "I feel that this condition [that is, the previously rated exposure to the working condition] is a burden for me". Respondents were prompted to rate the challenge and hindrance appraisals on a four-point rating format, ranging from "I do not agree at all" to "I fully agree". Similar single item measures for stressor appraisal were used by Webster et al. (2011). We chose however, a slightly different wording (Gerich 2016) with a stronger emphasis on the differentiation between challenge and hindrance.

Burnout was covered by the mean value given on the six items (for example, "How often do you feel tired?" or "How often are you emotionally exhausted?") of the Copenhagen Burnout Inventory (Kristensen et al. 2005), with five response categories (ranging from "never" to "always"). A Cronbach's alpha of 0.89 was revealed. 
Four items from Weyer et al.'s (1980) scale were used to measure work satisfaction (for example, "I have a truly interesting job") on a four-point rating format, ranging from "I do not agree at all" to "I fully agree" (Cronbach's alpha=0.73).

Four items from the short questionnaire for job analysis (Prümper et al. 1995) were used to cover social support from supervisors and colleagues (for example, "I can count on my supervisor when things are getting complicated" and "There is good cohesion between the members of our team/organization") on a four-point rating format ranging from "I do not agree at all" to "I fully agree" (Cronbach's alpha = 0.71).

Two out of three items taken from the short questionnaire for job analysis (Prümper et al. 1995) were available for the measurement of job control ("I can influence the work that I am assigned" and "I am able to plan and arrange my work independently") on a four-point rating format (ranging from "I do not agree at all" to "I fully agree"). Inter-item correlation was 0.43. The Spearman-Brown statistic, which is the most appropriate reliability coefficient for a two-item measure (Eisinga et al. 2013) is 0.61.

Descriptive information and zero-order correlations for all variables are given in Table 1.

The control variables were respondents' age, sex, and occupational status. These control variables were included based on results of previous research to avoid possible confounding effects. According to results shown by Doyle and Hind (2002), women for example, perceive higher stress but cope better than males. Moreover, Karasek and Theorell (1999) argue that women tend to work in lower occupational positions that are typically associated with lower job control. Also, work support was found to be a more important resource for men compared to women (Blanch and Aluja 2012). Strain was found to be more deleterious among young workers and for workers with low socioeconomic status (Kuper and Marmot 2003) and burnout was found to be more common among older workers with manual occupational status (Ahola et al. 2006).

\subsection{Statistical Analyses}

We used Mplus 7.4 (Muthén and Muthén 1998-2012) to test the moderated mediation models (Fig. 1). Separate analyses for the two outcomes (work satisfaction and burnout) were conducted. The models were estimated using a robust maximum likelihood estimation (MLR), with standard errors that are robust to the non-normality of observations and to the use of ordinal variables (Finney and DiStefano 2006). To control for missing data biases (the share of missing values on the study variables range from 0.2 to $10.6 \%$ ), we used a full-information MLR estimation (Enders 2010). Except for gender and occupational status, all variables were standardized prior to analysis and the computation of squared and product terms. Thus, throughout the paper we report standardized main, interaction and indirect effects (Cohen et al. 2003), which can also be interpreted as effect size measures (Ferguson 2009; MacKinnon 2008). To test multiple mediation, moderated mediation, and mediation including nonlinear effects, we followed the recommendation of Preacher and colleagues (Hayes and Preacher 2010; Preacher and Hayes 2008; Preacher et al. 2007). Bias-corrected bootstrapping with 5000 bootstrap samples was used to assess the significance of (conditional and instantaneous) indirect effects. Indirect effects are considered statistically significant if the 95\% confidence interval (CI) does not include 0 . 


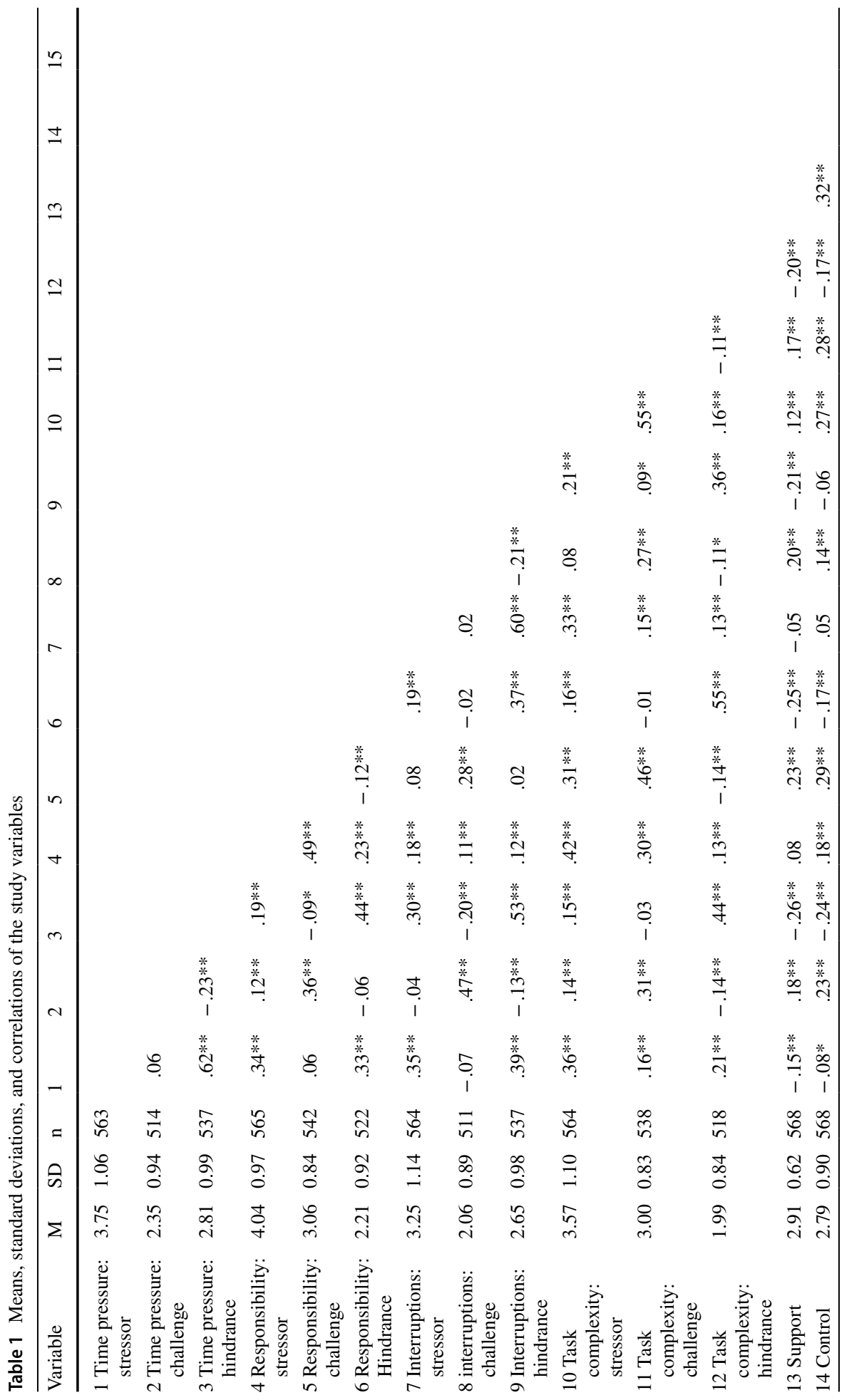




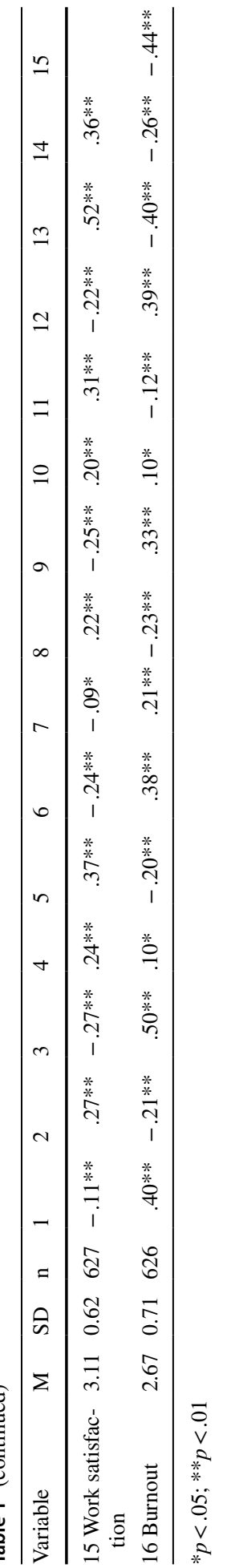




\section{Results}

The bivariate correlations of the study variables are reported in Table 1. All work demands are positively correlated with hindrance appraisal. Task complexity and responsibility are positively correlated with challenge appraisal, while time pressure and interruptions are not significantly correlated with challenge appraisal. The moderate negative correlations between challenge and hindrance appraisals for all demands ( $\mathrm{r}$ between -.11 and -.23 ) suggest that challenge and hindrance appraisals are not mutually exclusive. All challenge appraisals are positively correlated with work satisfaction and negatively correlated with burnout. All hindrance appraisals are negatively correlated with work satisfaction and positively correlated with burnout. Moreover, all work demands are correlated positively with burnout. However, whereas time pressure and interruptions are negatively correlated with work satisfaction, responsibility and task complexity are positively correlated to work satisfaction. Social support and job control are positively related to challenge appraisals and negatively related to hindrance appraisals (except for a nonsignificant correlation between job control and hindrance appraisal of interruptions). Finally, support and control are positively related to work satisfaction and negatively related to burnout.

Table 2 shows the results of the multivariate models. Detailed results with respect to simple slopes and conditional (instantaneous) indirect effects are presented in Table 3.

\subsection{Association of Work Demands with Challenge and Hindrance Appraisals (H1a, H1b)}

In Hypothesis H1a a curvilinear association between demand intensity and challenge appraisal is assumed. This is inspected by the effect of the squared demand intensity on challenge appraisal (Table 2, section I). As indicated by the significant effects of the squared work demands, curvilinear relationships between demand intensity and challenge appraisal are confirmed for three out of the four demands (task complexity, responsibility, time pressure). No significant relationship between demand intensity of interruptions and challenge appraisal is confirmed. Hence, Hypothesis $\mathrm{H} 1 \mathrm{a}$ is partially confirmed for three out of four demands. The shapes of these curvilinear associations are plotted in Fig. 2. Also, detailed information about the simple slopes at different regions of demand intensity is provided in Table 3. The type of the three significant associations between demand intensity and challenge appraisal could be described as a constant effect characteristic (according to the classification of Warr 1994). With respect to task complexity and responsibility, an increase in demand intensity is related to a significant increase in challenge appraisal for regions of low or medium demand intensity, which is attenuated (and not significant) for higher regions of demand intensity (Fig. 2a, b). Similarly, with respect to time pressure (Fig. 2c), there is a significant positive relationship between demand intensity and challenge appraisal at low levels of time pressure. This association becomes insignificant at higher levels of time pressure.

In Hypothesis $\mathrm{H} 1 \mathrm{~b}$, a linear relationship between demand intensity and hindrance appraisal is assumed. Therefore significant positive effects of the linear component of demand intensity but insignificant effects for the squared demand intensity are expected. The results (Table 2, section I) confirm significant linear positive associations between demand and hindrance appraisal for all four work demands. However, contrary to the expectation of $\mathrm{H} 1 \mathrm{~b}$, also a significant effect of the squared demand component is 


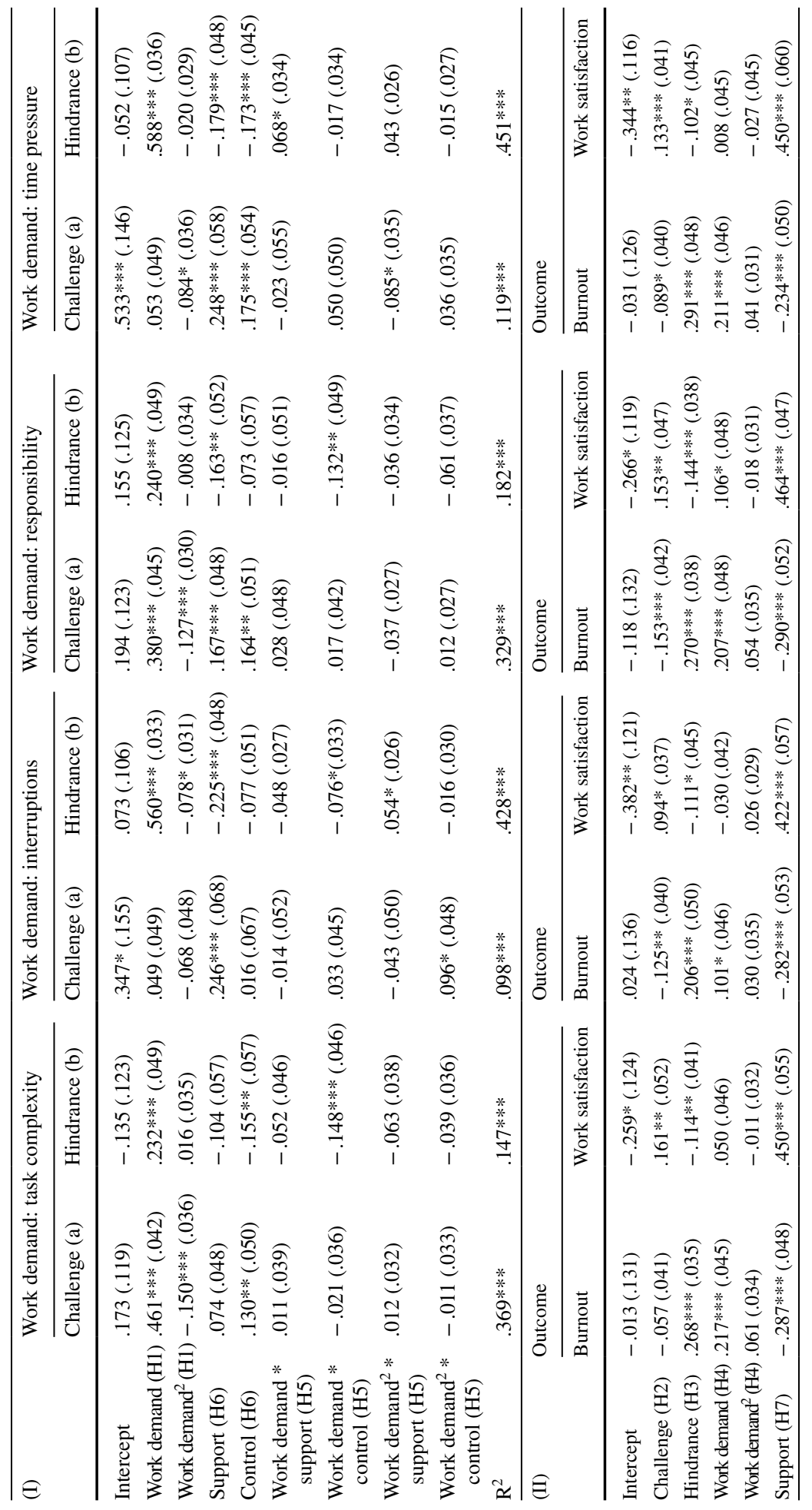




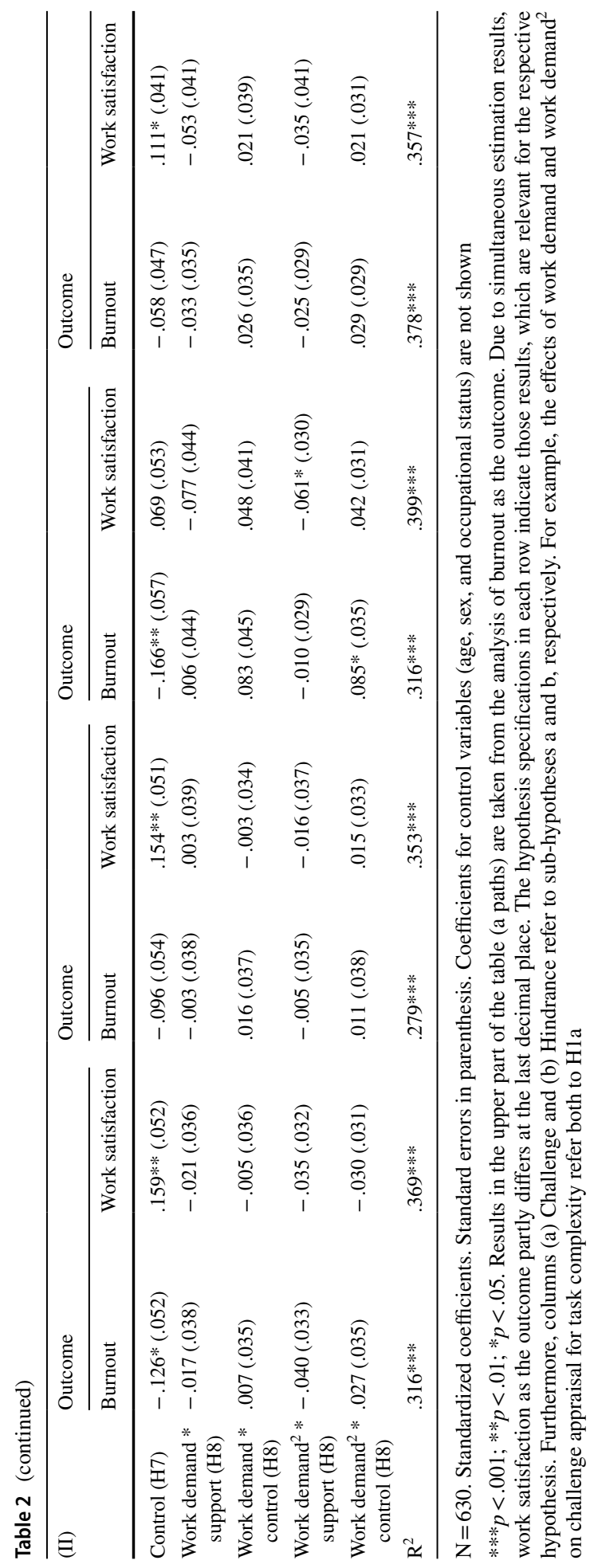


Table 3 Simple slopes and conditional (instantaneous) indirect effects

\begin{tabular}{|c|c|c|c|c|c|}
\hline & \multirow[t]{3}{*}{ Simple slopes } & \multicolumn{4}{|c|}{ Conditional (instantaneous) indirect effects } \\
\hline & & \multicolumn{2}{|c|}{ Outcome: Burnout } & \multicolumn{2}{|c|}{ Outcome: Work satisfaction } \\
\hline & & Estimates & $\mathrm{BC} 95 \% \mathrm{CI}$ & & $\mathrm{BC} 95 \% \mathrm{CI}$ \\
\hline \multicolumn{6}{|l|}{$T C^{2} \rightarrow$ Challenge } \\
\hline Low TC & $.761 * * *(.080)$ & -.043 & {$[-.112, .018]$} & $.122 *$ & {$[.049, .215]$} \\
\hline Mean TC & $.461 * * *(.042)$ & -.026 & {$[-.067, .011]$} & $.074 *$ & {$[.029, .128]$} \\
\hline High TC & $.161(.085)$ & -.009 & {$[-.037, .002]$} & $.026^{*}$ & {$[.002, .073]$} \\
\hline \multicolumn{6}{|l|}{$I N T^{2} \rightarrow$ Challenge } \\
\hline Low INT & $.185(.117)$ & -.023 & {$[-.068, .002]$} & .018 & {$[-.001, .058]$} \\
\hline Mean INT & $.049(.049)$ & -.006 & {$[-.022, .005]$} & .005 & {$[-.003, .019]$} \\
\hline High INT & $-.088(.097)$ & .011 & {$[-.011, .046]$} & -.008 & {$[-.037, .007]$} \\
\hline \multicolumn{6}{|l|}{$R E S^{2} \rightarrow$ Challenge } \\
\hline Low RES & $.633 * * *(.056)$ & $-.097 *$ & {$[-.160,-.043]$} & $.097 *$ & {$[.038, .163]$} \\
\hline Mean RES & $.380 * * *(.045)$ & $-.058^{*}$ & {$[-.100,-.026]$} & $.058 *$ & {$[.022, .103]$} \\
\hline High RES & $.126(.090)$ & -.019 & {$[-.061, .006]$} & .018 & {$[-.006, .063]$} \\
\hline \multicolumn{6}{|l|}{$T P^{2} \rightarrow$ Challenge } \\
\hline Low TP & $.220 * *(.067)$ & $-.020^{*}$ & {$[-.050,-.002]$} & $.030 *$ & {$[.010, .063]$} \\
\hline Mean TP & .053 (.049) & -.005 & {$[-.018, .003]$} & .007 & {$[-.004, .026]$} \\
\hline High TP & $-.115(.104)$ & .010 & {$[-.007, .043]$} & -.015 & {$[-.052, .010]$} \\
\hline$T C \rightarrow$ Hindrance & $.232 * * *(.049)$ & $.064 *$ & {$[.036, .099]$} & $-.026^{*}$ & {$[-.054,-.008]$} \\
\hline RES $\rightarrow$ Hindrance & $.240 * * *(.049)$ & $.065^{*}$ & {$[.036, .104]$} & $-.035^{*}$ & {$[-.062,-.016]$} \\
\hline$T P \rightarrow$ Hindrance & $.588 * * *(.036)$ & $.171^{*}$ & {$[.115, .234]$} & $-.060 *$ & {$[-.113,-.005]$} \\
\hline \multicolumn{6}{|c|}{$\mathrm{INT}^{2} \rightarrow$ Hindrance } \\
\hline Low INT & $.715 * * *(.069)$ & $.147 *$ & {$[.073, .231]$} & $-.080^{*}$ & {$[-.147,-.017]$} \\
\hline Mean INT & $.560 * * *(.033)$ & $.115^{*}$ & {$[.061, .177]$} & $-.062 *$ & {$[-.114,-.013]$} \\
\hline High INT & $.405 * * *(.072)$ & $.083 *$ & {$[.042, .141]$} & $-.045^{*}$ & {$[-.093,-.012]$} \\
\hline
\end{tabular}

TC task complexity, INT interruptions, RES responsibility, TP time pressure, Low values one SD below the mean, mean values at the mean, high values one SD above the mean, $C I$ confidence interval, $B C$ bias corrected. Standard errors in parentheses. $* * * p<.001 ; * * p<.01 ; * p<.05$. Asterisks beside the estimates of the indirect effects indicate that the $95 \%$ CI does not include 0

observed for interruptions, indicating a curvilinear relationship. Figure $2 \mathrm{~d}$ and simple slopes provided in Table 3 show that there is a monotonic positive association between interruptions and hindrance appraisal that flattens at high levels of interruptions, but remains positive and statistical significant across all levels of interruptions. Thus, for each of the four demands, higher demand intensity is related to higher hindrance appraisal. These results largely support $\mathrm{H} 1 \mathrm{~b}$ although a curvilinear (but monotonic) association between interruptions and hindrance appraisal is observed. 

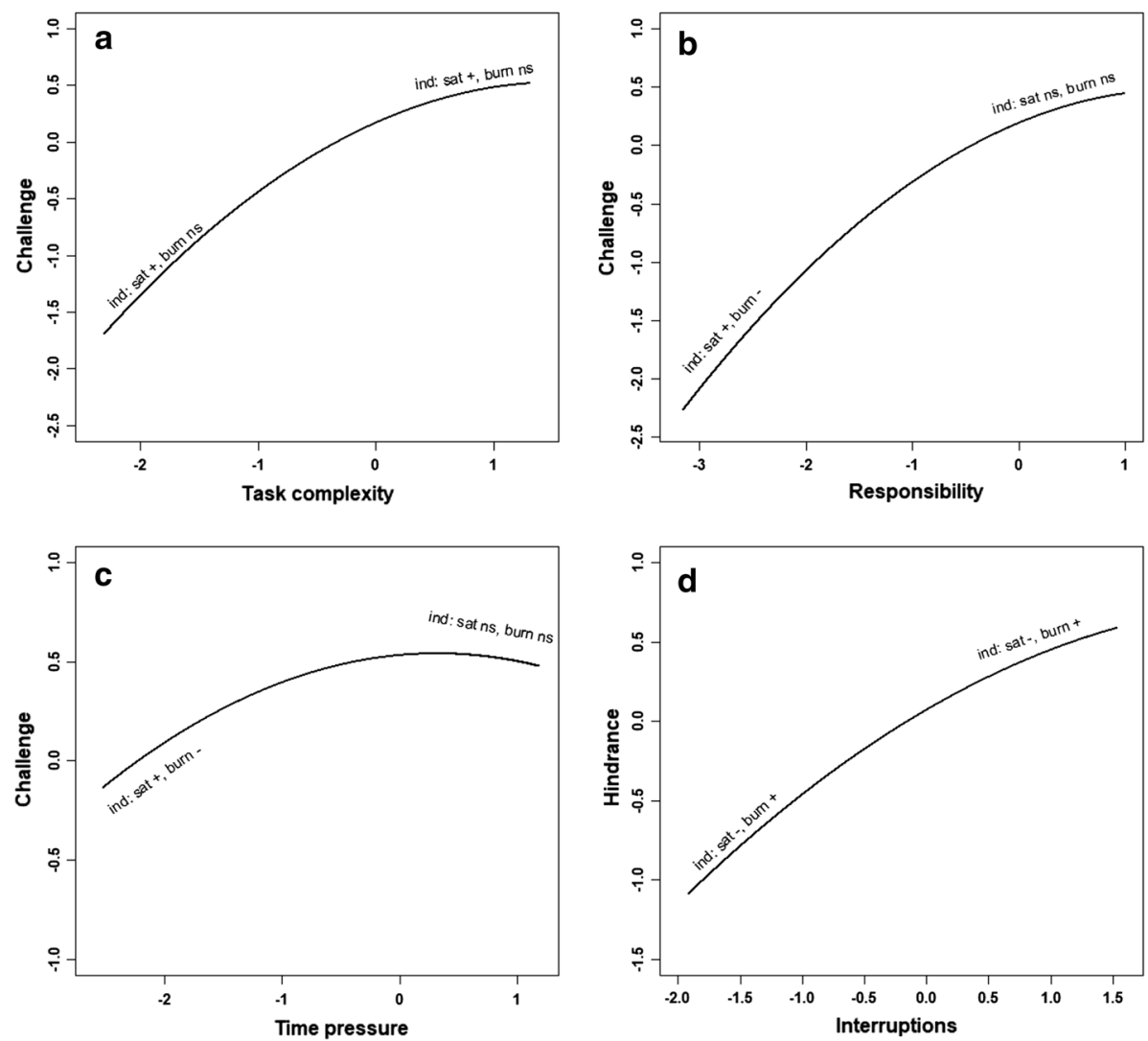

Fig. 2 Curvilinear associations between work demands and challenge and hindrance appraisal. Note ind direction of conditional (instantaneous) indirect effects ( $n s$ not significant) through challenge appraisal on work satisfaction (sat) and burnout (burn)

\subsection{Associations Between Appraisal and Outcome Variables (H2, H3)}

In Hypotheses $\mathrm{H} 2$ and $\mathrm{H} 3$ positive relationships of challenge appraisal, and negative associations of hindrance appraisal with work-satisfaction and health-related well-being are expected. As Table 2 (section II) shows, challenge appraisal is positively related and hindrance appraisal is negatively related to work satisfaction for all four demands. Hindrance appraisal is positively related to burnout for all four demands. Except for task complexity, challenge appraisal of demands is negatively related to burnout. Hence, H3 is supported for all four demands. H2 is supported for all four demands with respect to work satisfaction, but only partly supported with respect to burnout.

In sum however, it should be noted that challenge appraisals are more strongly associated with work satisfaction than with burnout, whereas hindrance appraisals are more strongly associated with burnout than work satisfaction. This is confirmed by additional analyses: the challenge appraisals of the four working conditions explain $18 \%$ of the variance of work satisfaction, but only $8 \%$ of the variance of burnout. By contrast, the 
hindrance appraisals of the four working conditions explain $30 \%$ of the variance of burnout, but only $12 \%$ of the variance of work satisfaction.

\subsection{Indirect Effects of Demands Through Appraisal on Outcome Variables (H4)}

With $\mathrm{H} 4$ it is expected that challenge and hindrance partially mediate the association between work demands and the two outcome variables. Indirect effects are shown in Table 3 and mapped in Fig. 2. There are significant indirect effects of demand intensity through challenge appraisal on work satisfaction for task complexity, responsibility and time pressure. However, due to the nonlinear association between demand intensity and challenge appraisal, these indirect effects depend on the level of demand intensity. In detail, the positive indirect effects of demand intensity on work satisfaction are attenuated and partly become insignificant at higher levels of work demands. Due to the insignificant association between demand intensity and challenge appraisal for interruptions, the indirect effects of interruptions on work satisfaction are also not significant. For two work demands (responsibility and time pressure), the same pattern of indirect effects (with opposite signs) is observed with respect to burnout.

In regard to the mediating role of hindrance appraisal, negative indirect effects on work satisfaction and positive indirect effects on burnout are confirmed for all four demands (Table 3). Although the association between interruptions and hindrance appraisal is curvilinear (but monotonic positive), the indirect effects are significant with constant direction across all levels of interruptions (see also Fig. 2d).

Furthermore, controlling for appraisal there are still direct positive linear effects of demand intensity on burnout for all four demands (Table 2, section II). With respect to work satisfaction, only a significant direct effect of responsibility is observed.

Taken together, there is full support for $\mathrm{H} 4$ concerning hindrance appraisal as a mediator between demand intensity and both outcome variables. H4 however, is only partly supported with respect to challenge appraisal as a mediator. The mediation role of challenge appraisal was confirmed for three out of four demands with respect to job satisfaction and for two out of four demands with respect to burnout. Moreover, as expected, all mediation effects regarding burnout are partial, because direct effects of work demands adjusted for appraisal are confirmed. In regard to work satisfaction as outcome variable, there are mainly indirect effects of work demands, which is in line with a full mediation hypothesis.

\subsection{Social Support and Job Control as Moderators of the Demand- Appraisal-Relationship (H5a, H5b)}

According to hypotheses $\mathrm{H} 5 \mathrm{a}$ and $\mathrm{H} 5 \mathrm{~b}$, interaction effects are expected, such that demands are more strongly appraised as challenge and less strongly appraised as hindrance under conditions of high job control and high social support. The tests for these interaction effects are show in Table 2, section I. The results show a significant interaction effect between the squared demand intensity of interruptions and job control on challenge appraisal. This effect is plotted in Fig. 3a. Under the condition of low job control, there is an inverted-ushaped association between demand intensity and challenge appraisal. In contrast, at high levels of job control the association between demand intensity and challenge appraisal is not significant, but challenge appraisal is on a constantly higher level compared to low job control. Thus, in line with H5a, interruptions are more strongly appraised as challenge 

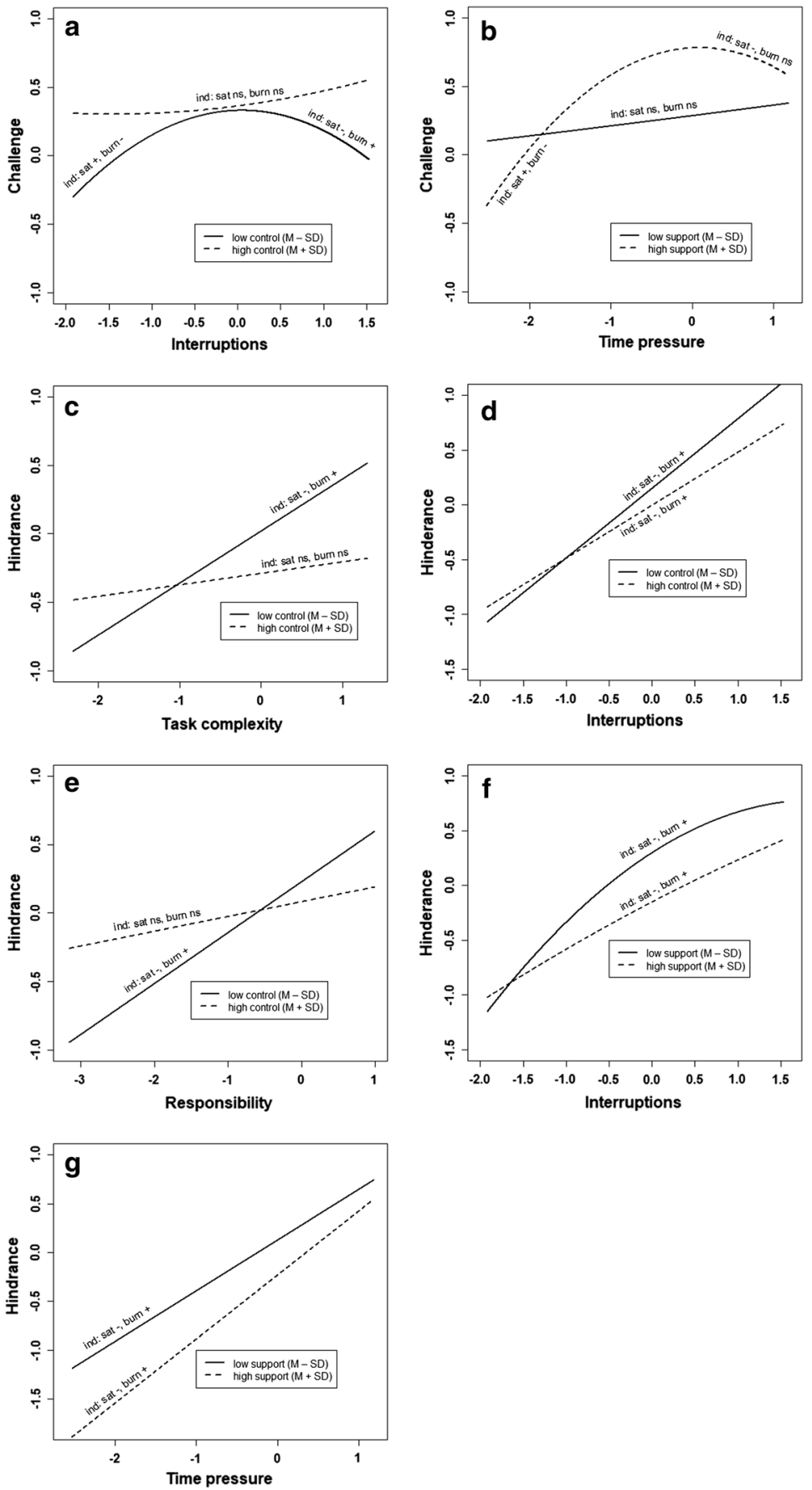

Fig. 3 Demand-appraisal-association moderated by social support and job control. Note ind direction of conditional (instantaneous) indirect effects ( $n s$ not significant) through hindrance appraisal on work satisfaction (sat) and burnout (burn) 
under the condition of high job control. With respect to time pressure, the relationship between demand and challenge appraisal is moderated by social support. Figure $3 \mathrm{~b}$ shows a non-significant positive association between demand intensity and challenge appraisal for individuals perceiving low social support. Under conditions of high social support, the association between time pressure and challenge appraisal turns to an inverted u-shaped curve. Thus, time pressure is most strongly appraised as challenge at mean levels of time pressure and high levels of social support. To sum up, these results provide some sparse support for H5a.

In regard to hindrance appraisal, interaction effects for all work demands with job control or social support are observed (Table 2, section I). As shown in Fig. 3c-e, the positive association of task complexity, interruptions, and responsibility with hindrance appraisal is amplified under conditions of low job control. Thus, these demands are most strongly appraised as hindrance under conditions of low job control. Similarly, the positive monotonic association between the intensity of interruptions and hindrance appraisal is amplified under conditions of low social support (Fig. 3f). Taken together, these results are in accordance with $\mathrm{H} 5 \mathrm{~b}$.

Conversely, the relationship between time pressure and hindrance appraisal is stronger under conditions of high social support than low social support. However, as seen in Fig. 3g, the level of hindrance appraisal is always lower for any value of time pressure under conditions of high social support than for low social support. To sum up, H5b is partly supported.

These moderator effects and the above-described mediation effects jointly support the assumption of a moderated mediation. As a consequence, the indirect effects of job demands through appraisal on the outcome variables depend on boundary conditions such as the amount of job control and social support, leading to even more complex conditional associations as could be seen from Fig. 3, where the conditional indirect effects are mapped (the full information about all conditional slopes and conditional indirect effects is provided in Online Resource 1). Especially the results show that the shapes of the curvilinear demand-challenge relationships depend on boundary conditions. Whereas the overall association between interruptions and challenge appraisal-and therefore also the indirect effects on the outcome variables - are not significant, the demand-challenge association turns to an inverted u-shaped relationship under conditions of low job control (Fig. 3a). Therefore, under conditions of low job control, a medium level of interruptions is associated with high challenge appraisal, whereas high and low levels of interruptions are associated with reduced challenge appraisal. This is also accompanied by conditional indirect effects on the outcome variables. Under conditions of low job control, an increase in interruptions at low levels is indirectly associated with a significant increase of job satisfaction and a decrease of burnout; further increases of interruptions at high levels are indirectly associated with a reduction of work satisfaction and an increase of burnout.

Similarly, the shape and indirect effects regarding time pressure depend on the perceived level of social support (Fig. 3b). Whereas the demand-challenge association regarding time pressure - and therefore also the indirect effects on the outcome variables - are not significant under conditions of low social support, the demand-challenge association turns to an inverted u-shaped relationship under conditions of high social support. Again this is accompanied by conditional indirect effects. Under conditions of high social support an increase of time pressure at low levels is indirectly associated with a significant increase of job satisfaction and a significant decrease of burnout, whereas increasing time pressure at high levels is indirectly associated with reduced job satisfaction and increased burnout. 


\subsection{Main Effects of Social Support and Job Control on Challenge and Hindrance Appraisal (H6a, H6b)}

With hypotheses H6a and H6b main effects of job resources (job control and social support) on challenge and hindrance appraisal adjusted for work demands are expected. Such main effects of job resources (job control or social support) on challenge and hindrance appraisal are confirmed for all demands (Table 2, section I). For three out of four demands, challenge appraisal is positively associated with job control (task complexity, responsibility, time pressure) and with social support (interruptions, responsibility, time pressure); this is in accordance with H6a.

Social support is negatively related to hindrance appraisal for three out of four demands (interruptions, responsibility, time pressure), and job control is negatively related to hindrance appraisal for two of the four demands (task complexity, time pressure), which is in accordance with H6b.

Therefore, partial support is found for H6a and H6b.

\subsection{Main Effects of Social Support and Job Control on Outcome Variables (H7)}

With H7 main effects of job resources (social support, job control) adjusted for demand intensity and appraisal on the two outcome variables are expected. The corresponding results in Table 2, section II confirm that social support is positively related to work satisfaction and negatively related to burnout in all models. Similarly, job control is positively related to work satisfaction (in three out of four models) and negatively related to burnout (in two out of four models). Hence, H7 is fully supported with respect to social support, and partially supported with respect to job control.

\subsection{Social Support and Job-Control as Moderators of the Demand-Outcome-Relationship (H8)}

Finally, Hypothesis H8 assumes interaction effects of demand intensity with job resources (job control and social support) with respect to the two outcome variables, as it would be expected by the JDC and JDR models. The results in Table 2, section II show that these interaction effects are not confirmed with respect to the linear terms. Only two significant interaction effects with the squared demand intensity of responsibility are observed with respect to work satisfaction and burnout. These results indicate that the curvilinear association between responsibility and burnout is moderated by control and that the curvilinear association between responsibility and work satisfaction is moderated by social support. Taken together, these results provide only weak support for H8. Moreover, the two interaction effects are only confirmed for the nonlinear association between demand and outcome variables, which is usually not considered in JDC and JDR models. 


\section{Discussion}

Within the classical challenge-hindrance framework (Cavanaugh et al. 2000), work stressors are a priori classified in hindrance or challenge stressors. This study shows that, contrary to a priori classifications of work stressors, work demands are simultaneously challenging and hindering. Aside from individual characteristics and resources, the level and balance of hindrance and challenge components embedded in a demanding situation depends on organizational and task-related boundary conditions such as the level of social support and job control provided. In our study, challenge components were positively related to work satisfaction and negatively related to burnout, whereas hindrance components were negatively associated with work satisfaction and positively related to burnout. With one exception (challenge appraisal of task complexity is not significantly related to burnout), these independent relationships between challenge and hindrance appraisal and outcome variables hold, even when adjusted for the intensity of the appraised work demands and other control variables (age, sex, and occupational status). With respect to the four work demands considered in our study (time pressure, interruptions, task complexity, and responsibility) however, challenge appraisals are more strongly associated with work satisfaction than with burnout, whereas hindrance appraisals are more strongly associated with burnout than with work satisfaction. Previous research with a priori classifications of challenge and hindrance stressors found that stressors categorized as challenge stressors are positively associated with favorable work attitudes, but negatively associated with healthrelated well-being (Boswell et al. 2004; LePine et al. 2004). In light of our findings, these results of previous research with a priori classifications of challenge stressors may reflect that, for some work demands, the positive effects of challenge components may outweigh the negative effect of hindrance components with respect to work attitudes, but also that the negative effects of hindrance components dominate the positive effects of challenge components with respect to health-related well-being.

Our results also confirmed some curvilinear relationships between demand intensity and appraisal. This was the case for challenge appraisal, but less so for hindrance appraisal, which was predominately linearly related to demand intensity.

With respect to task complexity, responsibility, and time pressure the type of curvilinear relationship with challenge appraisal predominately corresponds with Warr's (1994) vitamin CE (constant effects) heuristic. An increase in these job demands is appraised as more challenging up to a certain degree, but additional increments beyond this certain threshold do not entail additional benefits. As hindrance appraisal is linearly related to these work demands, an increase in hindrance appraisal induced by growth of demand intensity is also accompanied by an increase of challenge appraisal for low or moderate demand intensity. Hence, negative indirect effects on well-being through the hindrance component of the work demand are opposed by positive indirect effects through the challenge component for low or moderate levels of demand intensity. However, further intensification of high levels of work demands provokes additional hindrance appraisal that is no longer accompanied by additional challenge appraisal. Therefore, at the first look the demand-challenge association of these two work characteristics could suggest that an increase at high levels brings no additional benefit but also no disadvantages. This however, would ignore the detrimental effect of the constantly rising hindrance component.

Whether the negative effects through the hindrance component will exceed the (static) positive effects through the challenge component of high demand levels, to some extent depends on other boundary conditions, such as job control, which were found to buffer the 
demand-hindrance relationship for responsibility and task complexity. However, because the hindrance component is generally more relevant for burnout and the challenge component is more relevant for work satisfaction, the average association between these two demands (task complexity and responsibility) is positive for work satisfaction, but negative with respect to psychological wellbeing.

Moreover, our findings suggest that depending on boundary conditions - such as the perceived social support or job control-the type of demand-challenge relationships may also turn from Warr's vitamin CE to AD (additional decrement) characteristics. Such characteristics were found for work interruptions under conditions of low job control and for time pressure under conditions of high social support.

Under conditions of low job control, a medium level of interruptions is associated with high challenge appraisal, whereas lower and higher levels of interruptions are related to reduced challenge appraisal. Jett and George (2003) argued on the one hand that interruptions may conflict with employees' objectives and goal attainment, but on the other hand may also provide stimulation, distraction, and information acquisition, especially for routine tasks. Hence, consistent with our results, higher levels of interruptions are associated with an increase of the hindrance component. In addition however, a moderate level of interruptions may be stimulating under conditions of low autonomy, whereas the positive aspects disappear at high levels of interruptions, because employees do not have flexible opportunities to synchronize interruptions with their core work objectives.

The challenge appraisal of time pressure is not statistically related to the level of time pressure under conditions of low social support. By contrast, when social support is high, increasing levels of time pressure, up to a certain degree, are related to increasing challenge appraisal. The challenge appraisal however, declines again at high levels of time pressure. These results are in accordance with activation theory (Gardener and Cummings 1988), which suggests that excessively low levels of time pressure may provoke boredom, meaning that challenge appraisal grows when time pressure increases from low levels. However, positive affect will decrease again for overly high levels of time pressure due to reduced performance and overload.

The results suggest that time pressure and interruptions include positive challenge components under specific conditions. But, although the challenge component would contribute to job satisfaction stronger than the hindrance component, the bivariate correlation between both demands and job satisfaction is negative. This is found, because the relation between demand intensity and challenge appraisal is either insignificant or nonlinear (dependent on boundary conditions), while hindrance appraisal grows steadily with demand intensity. Therefore the positive effects of these demands on work satisfaction are slightly outweighed by the negative effects in sum.

As stated above, contrary to challenge appraisal, hindrance appraisal is mainly linearly related to the intensity of work demands. Therefore, higher demand intensity is associated with higher hindrance appraisal for all considered work demands. These results are in line with arguments brought forward by LePine et al. (2005), who argued that the idea that stressors could be beneficial up to some point seems plausible for challenge stressors, but not for hindrance stressors. In accordance with theories on job control, the hindrance appraisal of task complexity, interruptions, and responsibility is buffered by higher job control. Due to the indirect effects of demand intensity through hindrance appraisal on work attitudes and strain, job control also buffers these indirect negative effects on well-being. Less evidence was found for a buffering effect of social support on hindrance appraisal, except for the hindrance appraisal of interruptions. Consistent with theoretical expectations the strength of the positive relationship between the intensity of interruptions and 
hindrance appraisal is reduced under conditions of high versus low social support. With respect to time pressure, the positive relationship between demand intensity and hindrance appraisal is unexpectedly amplified with higher social support. However, further inspection (Fig. 3g) shows that although hindrance appraisal grows faster with increasing time pressure under conditions of high social support, the level of hindrance appraisal is always lower for any level of time pressure under conditions of high support than under low support. A possible interpretation of this is that social support helps reduce the hindering aspects of time pressure, but that the power of this buffering effect is reduced with increasing levels of time pressure. Therefore, social support may reduce the hindrance appraisal of low or medium time pressure, but not that of high time pressure.

Finally, even after controlling for challenge and hindrance appraisals, the direct effects of demand intensity on the two outcome variables remain. All four variables of demand intensity show significant positive effects on burnout. Based on these results, it can be concluded that a higher intensity of work demands promotes higher psychological strain, irrespective of the individual appraisal of the demanding situation. This result is in line with conclusions drawn from other research (Webster et al. 2011) in that working conditions can be harmful for health independently and regardless of individual estimations, although the direct effects of demand intensity are weaker than the direct effects of hindrance appraisal. On the contrary, only one significant direct effect of demand intensity on work satisfaction is observed (higher responsibility is related to higher work satisfaction), which is also weak compared to the direct effect of challenge and hindrance appraisals. Hence, it can be concluded that, unlike strain, it is predominately the individual appraisal of a specific demand intensity that is relevant for work satisfaction. Moreover, in contrast to challenge and hindrance appraisals, only weak evidence is found for interaction effects between social support and job control with demand intensity, although both significant interaction effects are consistent with theoretical expectations. First, a significant interaction effect exists between responsibility and job control with respect to burnout, such that the positive effect of responsibility on burnout is buffered by higher job control for medium levels of responsibility. Hence, the linear relationship between responsibility and burnout under conditions of low job control becomes a u-shaped relationship (in accordance with the vitamin AD characteristic) under conditions of high control. Second, the positive effect of responsibility on work satisfaction is amplified by higher social support.

The weak evidence for the interaction effects among job control, social support, and demand intensity is in line with results of previous research (Häusser et al. 2010). Moreover, both of the significant interaction effects of the study are only confirmed when curvilinear relationships are considered and when adjusted for the challenge and hindrance appraisals of work demands (both effects are insignificant when the challenge and hindrance appraisals are excluded from the path models). Nevertheless, the results show stronger evidence for the interaction effects between job resources (social support and job control) and demands with respect to challenge and hindrance appraisal than with respect to the demand-outcome relationship. Hence, in accordance with the suggestions of others (Mackey and Perrewé 2014), it can be concluded that these job resources more likely alter the perception of work demands than their direct effects on work attitudes and psychological strain.

This study has certain limitations. As the results are based on self-reported measures in a cross-sectional study, inflations of the associations between appraisal and outcome variables through common method bias are possible. As mentioned by Webster et al. (2011), among others, some findings (regarding differential associations with appraisal, mediation, and moderation effects) cannot be explained by common method bias; nevertheless, further 
research using objective measures of outcome variables is needed to validate the results. However, in a previous diary study among nurses, Johnston et al. (2016) confirmed that the experienced stress associated with different work tasks significantly predicted physiological measures of health (heart rate) even when adjusted for work task classification.

Moreover, due to the cross-sectional design of our study, the causality of the relationships between appraisal and the other variables is theoretically derived but not accessible for empirical testing. Maxwell and Cole (2007) pointed out that analyses of mediation processes that unfold over time by utilizing cross-sectional data have a high risk of generating biased estimates. As Preacher and Kelley (2011, p. 108) pointed out, "this criticism is valid, and similar criticism apply to any effect size measure based on analysis of crosssectional data when the process under study is a longitudinal one". On the other hand, similar to our study, Paškvan et al.'s (2015) longitudinal study among employees of a bank confirmed that work intensification at time 1 explained subsequent emotional exhaustion and work satisfaction at time 2 through hindrance appraisal of work intensification. Also, in a recently published time-lagged study with a 4-month time distance among airline employees, Liu and Li (2018) confirmed that challenge appraisal mediated the relationship between job complexity and work-related outcomes (work motivation and task persistence) and that hindrance appraisal mediated the relationship between role conflict and both outcomes. They also confirmed that the indirect effect of job complexity on work motivation through challenge appraisal was moderated by respondents' task efficacy. Hence, although replications of our presented results with longitudinal data are clearly necessary, previous longitudinal research has confirmed the fundamental causal assumptions of our cross-sectional research.

Aside from causality, the presented research shows that the same stressor intensity may be appraised differently by employees and this difference likely depends on other (often unknown) boundary conditions of the organizational setting. Moreover, after accounting for stressor intensity, it was found that stressor appraisal explains unique variance in outcome variables measured at the same time point. Hence, instead of inferring the relevance of work stressors from a priori categorizations, it is recommended to consider some kind of appraisal measures in psychosocial risk assessments, especially when these assessments are used to draw inferences about possible psychosocial hazards of workplaces.

Furthermore, due to limited space in the questionnaire, we only used single-item measures for challenge and hindrance appraisal and a two-item measure for job control. Future research could use multiple item measures for challenge and hindrance appraisal, such as those developed by Searle and Auton (2015), and a more comprehensive measure for job control to further validate the presented findings.

Finally, we tested complex moderated mediation models including linear-by-linear interactions, curvilinear effects as well as curvilinear-by-linear interactions. Procedures for detecting interaction effects are generally plagued with low statistical power (Shieh 2009). The problem of low power becomes even more pronounced when curvilinear effects and interaction effects are tested simultaneously (Moosbrugger et al. 2009). Therefore, it might be difficult to replicate our findings-especially the complex curvilinear-by-linear interaction effects-in future research (Anderson and Maxwell 2017). In order to guide future research, we conducted a power analysis using a Monte Carlo simulation (Thoemmes et al. 2010). We first estimated the power of our models given the sample size of $n=630$. We then determined the sample size needed for adequate power (0.80). Although critically discussed in the literature (Anderson and Maxwell 2017), we used the results reported within this paper as starting values for the estimated (true) population effects. Following Cohen (1992), we categorized the reported effects as "no effect", "very small effect" (uniquely 
explained variance $(\mathrm{uev})=.01)$, "small effect" (uev=.02), "small to moderate effect" (uev=.078), "moderate effect" (uev=.13), "moderate to strong effect" (uev=.2), and "strong effect" (uev =.259). To avoid a power overestimation due to a potential overestimation of the effects within this study, we assumed lower values (that is, one effect size category below the initial category) for the curvilinear and interaction population effects. This strategy largely coincides with the safeguard power approach advocated by Perugini et al. (2014). We estimated two different models. The first one mimics the results for responsibility and task complexity and includes curvilinear effects of work demand on challenge appraisal and interaction effects of job control and work demand on hindrance appraisal. The second model corresponds to the results for time pressure and interruptions, which simultaneously includes curvilinear-by-linear interactions of work demand and control and support, respectively. Results show that our study has high power (.97) to detect curvilinear effects, but low power to detect linear-by-linear as well as curvilinear-by-linear interaction effects (ranging from .28 to .63). In order to have sufficient power to detect the linear-bylinear interaction effect, as well as the very small curvilinear-by-linear interactions, a sample size of $n>2.000$ would be needed.

\section{Conclusion}

Despite the limitations mentioned above, this research suggests that the individual evaluation of job demands is an important mediating path between job demands and their effects on work attitudes and psychological strain. The results show that organizational properties such as job control and social support alter these perceptions and, thus, the way that job demands affect well-being. Our research supports the presumption of others that the appraisal and the consequences of job demands may depend on properties of the occupational setting (Bakker 2014; Bakker and Demerouti 2017). Therefore, we agree with the recommendations raised in prior research that additional research for a more complete understanding of the stressor-appraisal process and the associated organizational moderators is needed (Bakker and Demerouti 2017; Demerouti and Bakker 2011; LePine et al. 2016; Mackey and Perrewé 2014; Spector, 2002). The presented results show that some assumptions of established theoretical frameworks - such as the interaction or buffer effects of job resources-for which previous research only found weak evidence-may only be identified when subjective appraisals of job characteristics are included. Consequently, established theoretical frameworks such as the job demand control or the job demand resources model could be refined by including challenge and hindrance appraisals of the proposed demands and resources, rather than inferring their nature from a priori classifications. Moreover, the results confirm that some job demands contain both challenging and hindering components, which were shown to independently affect work attitudes and strain in opposite ways. The balance between both components is variable and likely dependent on other boundary conditions of the organizational setting. The results further suggest that the challenge component is more influential for work attitudes, whereas the hindrance component is more influential for psychological strain. From a practical perspective, workplace interventions that aim to reduce or enhance the intensity of work demands based on a priori categorizations should be treated with care. Although reducing some work demands would probably reduce an associated hindering component and therefore reduce psychological strain, it may also reduce an associated challenge component that may, in turn, reduce work satisfaction and motivation. Conversely, interventions to further enhance work demands 
that are assumed to be challenging may provoke additional detrimental effects when the nonlinearity of challenge components is ignored. Due to the complexity of the nonlinear relationships between the challenge and hindrance components and demand intensity, which are dependent on various organizational properties, generalized a priori categorizations of work demands should be avoided. Hence, organizational interventions as well as the evaluation of such interventions should consider the subjective challenge and hindrance appraisals of employees. As a practical implication, risk inventories that are used in workplace health promotion should include measures of appraisal to guide and evaluate interventions that are aimed to reduce hindering components and to maximize challenge components of various job characteristics.

Acknowledgements Open access funding provided by Johannes Kepler University Linz. This research was supported by the Upper Austrian Sickness Fund.

Open Access This article is distributed under the terms of the Creative Commons Attribution 4.0 International License (http://creativecommons.org/licenses/by/4.0/), which permits unrestricted use, distribution, and reproduction in any medium, provided you give appropriate credit to the original author(s) and the source, provide a link to the Creative Commons license, and indicate if changes were made.

\section{References}

Ahola, K., Honkonen, T., Kivimäki, M., Virtanen, M., Isometsä, E., Aromaa, A., et al. (2006). Contribution of burnout to the association between job strain and depression: The health 2000 study. Journal of Occupational and Environmental Medicine. https://doi.org/10.1097/01.jom.0000237437.84513.92.

Anderson, S. F., \& Maxwell, S. E. (2017). Addressing the "replication crisis": Using original studies to design replication studies with appropriate statistical power. Multivariate Behavioral Research, 52(3), 305-324.

Bakker, A. B. (2014). Daily fluctuations in work engagement. European Psychologist, 19(4), 227-236. https ://doi.org/10.1027/1016-9040/a000160.

Bakker, A. B., \& Demerouti, E. (2007). The Job Demands-Resources model: State of the art. Journal of Managerial Psychology, 22(3), 309-328. https://doi.org/10.1108/02683940710733115.

Bakker, A. B., \& Demerouti, E. (2017). Job demands-resources theory: Taking stock and looking forward. Journal of Occupational Health Psychology. https://doi.org/10.1037/ocp0000056.

Blanch, A., \& Aluja, A. (2012). Social support (family and supervisor), work-family conflict, and burnout: Sex differences. Human Relations. https://doi.org/10.1177/0018726712440471.

Boswell, W. R., Olson-Buchanan, J. B., \& LePine, M. A. (2004). Relations between stress and work outcomes: The role of felt challenge, job control, and psychological strain. Journal of Vocational Behavior, 64(1), 165-181. https://doi.org/10.1016/S0001-8791(03)00049-6.

Cavanaugh, M. A., Boswell, W. R., Roehling, M. V., \& Boudreau, J. W. (2000). An empirical examination of self-reported work stress among US managers. Journal of Applied Psychology, 85(1), 65-74. https ://doi.org/10.1037/0021-9010.85.1.65.

Chong, D. S. F., van Eerde, W., Chai, K. H., \& Rutte, C. G. (2011). A double-edged sword: The effects of challenge and hindrance time pressure on new product development teams. IEEE Transactions on Engineering Management, 58(1), 71-86. https://doi.org/10.1109/TEM.2010.2048914.

Cohen, J. (1992). A power primer. Psychological Bulletin, 112(1), 155-159.

Cohen, J., Cohen, P., West, S. G., \& Aiken, L. S. (2003). Applied multiple regression/correlation analysis for the behavioral sciences (3rd ed.). Mahwah, NJ: Erlbaum.

Demerouti, E., \& Bakker, A. B. (2011). The Job Demands-Resources model: Challenges for future research. SA Journal of Industrial Psychology, 37(2), 1-9. https://doi.org/10.4102/sajip.v37i2.974.

Doyle, C., \& Hind, P. (2002). Occupational stress, burnout and job status in female academics. Gender, Work \& Organization. https://doi.org/10.1111/1468-0432.00047.

Eisinga, R., te Grotehhuis, M., \& Pelzer, B. (2013). The reliability of a two-item scale: Pearson, Cronbach or Spearman-Brown? International Journal of Public Health. https://doi.org/10.1007/s0003 8-012-0416-3. 
Enders, C. K. (2010). Applied missing data analysis: Methodology in the social sciences. New York: Guilford Press.

Ferguson, C. J. (2009). An effect size primer: A guide for clinicians and researchers. Professional Psychology: Research and Practice, 40(5), 532-538.

Finney, S. J., \& DiStefano, C. (2006). Non-normal and categorical data in structural equation modeling. In G. R. Hancock \& R. O. Mueller (Eds.), Quantitative methods in education and the behavioral sciences. Structural equation modeling: A second course (pp. 269-314). Greenwich, CT: IAP.

Ganster, D. C., \& Rosen, C. C. (2013). Work stress and employee health: A multidisciplinary review. Journal of Management, 39(5), 1085-1122. https://doi.org/10.1177/0149206313475815.

Gardener, D. G., \& Cummings, L. L. (1988). Activation theory and job design: Review and reconceptualization. In B. M. Staw \& L. L. Cummings (Eds.), Research in organizational behavior (Vol. 10, pp. 81-122). Greenwich: JAI Press.

Gerich, J. (2016). The relevance of challenge and hindrance appraisals of working conditions for employees' health. International Journal of Stress Management. https://doi.org/10.1037/str0000038.

Gomes, A. R., Faria, S., \& Lopes, H. (2016). Stress and psychological health: Testing the mediating role of cognitive appraisal. Western Journal of Nursing Research. https://doi.org/10.1177/019394591665466 6.

Häusser, J. A., Mojzisch, A., Niesel, M., \& Schulz-Hardt, S. (2010). Ten years on: A review of recent research on the Job Demand-Control (-Support) model and psychological well-being. Work \& Stress, 24(1), 1-35. https://doi.org/10.1080/02678371003683747.

Hayes, A. F., \& Preacher, K. J. (2010). Quantifying and testing indirect effects in simple mediation models when the constituent paths are nonlinear. Multivariate Behavioral Research, 45(4), 627-660. https:// doi.org/10.1080/00273171.2010.498290.

Jett, Q. R., \& George, J. M. (2003). Work interrupted: A closer look at the role of interruptions in organizational life. Academy of Management Review, 28(3), 494-507. https://doi.org/10.5465/ AMR.2003.10196791.

Johnson, J. V., \& Hall, E. M. (1988). Job strain, work place social support, and cardiovascular disease: A cross-sectional study of a random sample of the Swedish working population. American Journal of Public Health, 78(10), 1336-1342.

Johnston, D., Bell, C., Jones, M., Farquharson, B., Allan, J., Schofield, P., et al. (2016). Stressors, appraisal of stressors, experienced stress and cardiac response: A real-time, real-life investigation of work stress in nurses. Annals of Behavioral Medicine, 50(2), 187-197. https://doi.org/10.1007/s12160-015-9746-8.

Karasek, R. A. (1979). Job demands, job decision latitude, and mental strain: Implications for job redesign. Administrative Science Quarterly, 24(2), 285. https://doi.org/10.2307/2392498.

Karasek, R., \& Theorell, T. (1999). Healthy work: Stress, productivity, and the reconstruction of working life (6th ed.). New York, NY: Basic Books.

Kristensen, T. S., Borritz, M., Villadsen, E., \& Christensen, K. B. (2005). The Copenhagen Burnout Inventory: A new tool for the assessment of burnout. Work \& Stress, 19(3), 192-207. https://doi. org/10.1080/02678370500297720.

Kuper, H., \& Marmot, M. (2003). Job strain, job demands, decision latitude, and risk of coronary heart disease within the Whitehall II study. Journal of Epidemiology and Community Health. https://doi. org/10.1136/jech.57.2.147.

LePine, J. A., LePine, M. A., \& Jackson, C. L. (2004). Challenge and hindrance stress: Relationships with exhaustion, motivation to learn, and learning performance. The Journal of Applied Psychology, 89(5), 883-891. https://doi.org/10.1037/0021-9010.89.5.883.

LePine, J. A., Podsakoff, N. P., \& LePine, M. A. (2005). A meta-analytic test of the challenge stressor-hindrance stressor framework: An explanation for inconsistent relationships among stressors and performance. Academy of Management Journal. https://doi.org/10.5465/amj.2005.18803921.

LePine, M. A., Zhang, Y., Crawford, E. R., \& Rich, B. L. (2016). Turning their pain to gain: Charismatic leader influence on follower stress appraisal and job performance. Academy of Management Journal, 59(3), 1036-1059. https://doi.org/10.5465/amj.2013.0778.

Leung, K., Huang, K.-L., Su, C.-H., \& Lu, L. (2011). Curvilinear relationships between role stress and innovative performance: Moderating effects of perceived support for innovation. Journal of Occupational and Organizational Psychology. https://doi.org/10.1348/096317910X520421.

Liu, C., \& Li, H. (2018). Stressors and stressor appraisals: The moderating effect of task efficacy. Journal of Business and Psychology. https://doi.org/10.1007/s10869-016-9483-4.

Mackey, J. D., \& Perrewé, P. L. (2014). The AAA (appraisals, attributions, adaption) model of job stress: The critical role of self-regulation. Organizational Psychology Review, 4(3), 258-278.

MacKinnon, D. P. (2008). Introduction to statistical mediation analysis. Mahwah, NJ: Erlbaum. 
Maxwell, S. E., \& Cole, D. A. (2007). Bias in cross-sectional analyses of longitudinal mediation. Psychological Methods. https://doi.org/10.1037/1082-989X.12.1.23.

Meyerding, S. G. H. (2015). Job characteristics and job satisfaction: A test of Warr's vitamin model in German horticulture. The Psychologist-Manager Journal. https://doi.org/10.1037/mgr0000029.

Moosbrugger, H., Schermelleh-Engel, K., Kelava, A., \& Klein, A. G. (2009). Testing multiple nonlinear effects in structural equation modeling: A comparison of alternative estimation approaches. In T. Teo \& M. S. Khine (Eds.), Structural equation modelling in educational research: Concepts and applications (pp. 103-136). Rotterdam, NL: Sense Publishers.

Muthén, L. K., \& Muthén, B. O. (1998-2012). Mplus user's guide (2nd ed.). Los Angeles, CA: Muthén \& Muthén.

Paškvan, M., Kubicek, B., Prem, R., \& Korunka, C. (2015). Cognitive appraisal of work intensification. International Journal of Stress Management. https://doi.org/10.1037/a0039689.

Pearsall, M. J., Ellis, A. P., \& Stein, J. H. (2009). Coping with challenge and hindrance stressors in teams: Behavioral, cognitive, and affective outcomes. Organizational Behavior and Human Decision Processes, 109(1), 18-28. https://doi.org/10.1016/j.obhdp.2009.02.002.

Perugini, M., Gallucci, M., \& Costantini, G. (2014). Safeguard power as a protection against imprecise power estimates. Perspectives on Psychological Science, 9(3), 319-332.

Podsakoff, N. P., LePine, J. A., \& LePine, M. A. (2007). Differential challenge stressor-hindrance stressor relationships with job attitudes, turnover intentions, turnover, and withdrawal behavior: A meta-analysis. The Journal of Applied Psychology, 92(2), 438-454. https://doi.org/10.1037/0021-9010.92.2.438.

Preacher, K. J., \& Hayes, A. F. (2008). Asymptotic and resampling strategies for assessing and comparing indirect effects in multiple mediator models. Behavior Research Methods, 40(3), 879-891.

Preacher, K. J., \& Kelley, K. (2011). Effect size measures for mediation models: Quantitative strategies for communicating indirect effects. Psychological Methods. https://doi.org/10.1037/a0022658.

Preacher, K. J., Rucker, D. D., \& Hayes, A. F. (2007). Addressing moderated mediation hypotheses: Theory, methods, and prescriptions. Multivariate Behavioral Research, 42(1), 185-227. https://doi. org/10.1080/00273170701341316.

Preston, M. S. (2018). Does job control moderate work demands' nonlinear association with job strain?: A two-sample study using human service case managers. Human Service Organizations: Management, Leadership \& Governance. https://doi.org/10.1080/23303131.2018.1457582.

Prümper, J., Hartmannsgruber, K., \& Frese, M. (1995). KFZA. Kurz-Fragebogen zur Arbeitsanalyse. Zeitschrift für Arbeits- und Organisationspsychologie, 39(3), 125-132.

Reis, D., Hoppe, A., Arndt, C., \& Lischetzke, T. (2016). Time pressure with state vigour and state absorption: Are they non-linearly related? European Journal of Work and Organizational Psychology, 26(1), 94-106. https://doi.org/10.1080/1359432X.2016.1224232.

Searle, B. J., \& Auton, J. C. (2015). The merits of measuring challenge and hindrance appraisals. Anxiety Stress and Coping, 28(2), 121-143. https://doi.org/10.1080/10615806.2014.931378.

Selye, H. (1974). Stress without distress (1st ed.). Philadelphia: Lippincott.

Shieh, G. (2009). Detecting interaction effects in moderated multiple regression with continuous variables power and sample size considerations. Organizational Research Methods, 12(3), 510-528.

Sonnentag, S., \& Frese, M. (2003). Stress in organizations. In W. C. Borman, D. R. Ilgen, \& R. J. Klimoski (Eds.), Handbook of psychology. Industrial and organizational psychology (Vol. 12, pp. 453-491). Hoboken, New Jersey: Wiley.

Spector, P. E. (2002). Employee control and occupational stress. Current Directions in Psychological Science, 11(4), 133-136. https://doi.org/10.1111/1467-8721.00185.

Thoemmes, F., MacKinnon, D. P., \& Reiser, M. R. (2010). Power analysis for complex mediational designs using Monte Carlo methods. Structural Equation Modeling, 17(3), 510-534.

Warr, P. (1994). A conceptual framework for the study of work and mental health. Work \& Stress, 8(2), 84-97. https://doi.org/10.1080/02678379408259982.

Warr, P. (2014). Environmental "vitamins", personal judgments, work values, and happiness. In S. Cartwright \& C. L. Cooper (Eds.), The Oxford handbook of organizational well-being (pp. 57-85). Oxford, GB: Oxford University Press.

Webster, J. R., Beehr, T. A., \& Love, K. (2011). Extending the challenge-hindrance model of occupational stress: The role of appraisal. Journal of Vocational Behavior, 79(2), 505-516. https://doi.org/10.1016/j. jvb.2011.02.001.

Weyer, G., Hodapp, V., \& Neuhauser, S. (1980). Weiterentwicklung von Fragebogenskalen zur Erfassung der subjektiven Belastung und Unzufriedenheit im beruflichen Bereich (SBUS-B). Psychologische Beitrage, 22(2), 335-355. 
Widmer, P. S., Semmer, N. K., Kälin, W., Jacobshagen, N., \& Meier, L. L. (2012). The ambivalence of challenge stressors: Time pressure associated with both negative and positive well-being. Journal of Vocational Behavior, 80, 422-433. https://doi.org/10.1016/j.jvb.2011.09.006.

Zoupanou, Z. (2015). Being work interrupted: A qualitative study of office managers. International Journal of Quantitative and Qualitative Research Methods, 3(1), 1-24.

Publisher's Note Springer Nature remains neutral with regard to jurisdictional claims in published maps and institutional affiliations. 